\title{
Gyrotron multistage depressed collector based on $E \times B$ drift concept using azimuthal electric field. I. Basic design
}

Cite as: Phys. Plasmas 25, 033108 (2018); https://doi.org/10.1063/1.5016296

Submitted: 17 November 2017 . Accepted: 20 February 2018 . Published Online: 14 March 2018

Chuanren Wu (吴传人) (D), Ioannis Gr. Pagonakis, Konstantinos A. Avramidis (D), Gerd Gantenbein, Stefan Illy, Manfred Thumm (D), and John Jelonnek (D)

ARTICLES YOU MAY BE INTERESTED IN

Conceptual designs of E $\times$ B multistage depressed collectors for gyrotrons

Physics of Plasmas 24, 043102 (2017); https://doi.org/10.1063/1.4979291

Multistage depressed collector conceptual design for thin magnetically confined electron beams

Physics of Plasmas 23, 043114 (2016); https://doi.org/10.1063/1.4947565

Investigation on heat transfer analysis and its effect on a multi-mode, beam-wave interaction for a $140 \mathrm{GHz}, \mathrm{MW}$-class gyrotron

Physics of Plasmas 25, 043101 (2018); https://doi.org/10.1063/1.4996701 


\title{
Gyrotron multistage depressed collector based on $E \times B$ drift concept using azimuthal electric field. I. Basic design
}

\author{
Chuanren Wu (吴传人), Ioannis Gr. Pagonakis, Konstantinos A. Avramidis, \\ Gerd Gantenbein, Stefan Illy, Manfred Thumm, and John Jelonnek \\ Institute for Pulsed Power and Microwave Technology, Karlsruhe Institute of Technology, \\ Eggenstein-Leopoldshafen D-76344, Germany
}

(Received 17 November 2017; accepted 20 February 2018; published online 14 March 2018)

\begin{abstract}
Multistage Depressed Collectors (MDCs) are widely used in vacuum tubes to regain energy from the depleted electron beam. However, the design of an MDC for gyrotrons, especially for those deployed in fusion experiments and future power plants, is not trivial. Since gyrotrons require relatively high magnetic fields, their hollow annular electron beam is magnetically confined in the collector. In such a moderate magnetic field, the MDC concept based on $\boldsymbol{E} \times \boldsymbol{B}$ drift is very promising. Several concrete design approaches based on the $\boldsymbol{E} \times \boldsymbol{B}$ concept have been proposed. This paper presents a realizable design of a two-stage depressed collector based on the $\boldsymbol{E} \times \boldsymbol{B}$ concept. A collector efficiency of $77 \%$ is achievable, which will be able to increase the total gyrotron efficiency from currently $50 \%$ to more than $60 \%$. Secondary electrons reduce the efficiency only by $1 \%$. Moreover, the collector efficiency is resilient to the change of beam current (i.e., space charge repulsion) and beam misalignment as well as magnetic field perturbations. Therefore, compared to other $\boldsymbol{E} \times \boldsymbol{B}$ conceptual designs, this design approach is promising and fairly feasible.

https://doi.org/10.1063/1.5016296
\end{abstract}

\section{INTRODUCTION}

Gyrotrons are microwave sources for electron cyclotron heating and current drive in fusion experiments. Electrons in a gyrotron are accelerated by a high voltage, which can be, e.g., $80 \mathrm{kV}$ in the ITER gyrotron. ${ }^{1}$ Usually less than $35 \%$ of the electron kinetic energy is converted into microwaves, while the remainder of the input electric energy remains in the spent electron beam. Depressed collectors can recover a part of this energy and thus increase the gyrotron efficiency. SingleStage Depressed Collectors (SDCs) being used in fusion gyrotrons $^{2-4}$ usually have $50 \%$ to $60 \%$ collector efficiency $\left(\eta_{\text {col }}=P_{\text {recovered }} / P_{\text {spent beam }}\right)$, which corresponds to an overall gyrotron efficiency $\left(\eta_{\text {total }}\right)$ of up to approximately $50 \%$. However, for a gyrotron generating megawatts of continuous RF power, plenty of spent beam energy is converted into heat on the water-cooled collector. Taking other losses in a gyrotron into account, to accomplish the $\eta_{\text {total }}>60 \%$ goal of efficiency, the collector efficiency should be $\eta_{\text {col }}>74 \%,{ }^{5}$ which is beyond the capability of an SDC. In order to achieve this goal, the depressed collector should be extended to multiple stages, the so-called Multistage Depressed Collector (MDC).

MDCs have been widely applied in other vacuum tubes, especially in the traveling wave tubes where high efficiency is demanded. ${ }^{6,7}$ However, to the best knowledge of the authors, no experiment on any gyrotron MDC has been published yet. There are several reasons that cause the design of a gyrotron MDC to be non-trivial. First, there is still a strong magnetic field in the gyrotron collector region, which is above $100 \mathrm{mT}$ at the collector entrance. The field remains at tens of $\mathrm{mT}$ even after a $1 \mathrm{~m}$ axial offset in the collector. Sorting electrons in such a strong magnetic field is difficult. Moreover, the impact area on the electrodes is limited by the bundle of the electron beam, which is determined by the magnetic confinement. The straightforward solution targeting the magnetic field is to weaken it to approximately $10 \mathrm{mT}$, which will reduce the confinement. The demagnetization requires a large volume. A nonadiabatic transition of the magnetic field helps to reduce the required space and can further spread the electron beam spatially. ${ }^{8-10}$ Second, fusion gyrotrons have annular electron beams with small orbits. If the electric and magnetic scalar potentials are axisymmetric, the magnetic flux enclosed in the electron beam is constant (Busch theorem). In order to reduce the local magnetic field without increasing the collector size a lot, additional coils or pole pieces have to be inserted near the axis in the conventional approach. ${ }^{5,8,9}$ This is inconvenient. Third, trajectories of secondary electrons (including the elastically and inelastically reflected ones) are hard to control concerning the influence of both the electric and magnetic fields.

Aside from the conventional nonadiabatic approach described above, another gyrotron MDC concept based on the $\boldsymbol{E} \times \boldsymbol{B}$ drift has been proposed. ${ }^{11}$ This concept makes use of the $\boldsymbol{E} \times \boldsymbol{B}$ drift to perfectly sort and distribute electrons, while the same drift also prevents the secondary electrons being returned. Therefore, this concept seems to be very promising. Three kinds of MDC design approaches based on the $\boldsymbol{E} \times \boldsymbol{B}$ concept have been proposed, ${ }^{12}$ where the drift can be planar or radial, depending on the orientation of the fields. For the planar drift case, the hollow annular electron beam is transformed first into one or more sheet beams. Then, the $\boldsymbol{E} \times \boldsymbol{B}$ drift is applied on the sheet beams. ${ }^{13,14}$ In the radial drift approaches, there will always be a longitudinal component of the electric field to decelerate the electrons, with a longitudinal component of the magnetic field retained from the gyrotron magnet. The azimuthal electric field produced by pairs of helical electrodes ${ }^{11,15}$ can cause radial drifts. Alternatively, the azimuthal component of the magnetic field 


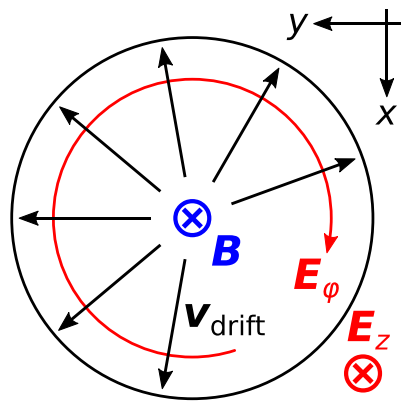

FIG. 1. Sketch of the field components and the drift.

induced by a special type of collector coil can also create radial drifts. ${ }^{16}$

Among the various MDC ideas, the azimuthal electric field approach ${ }^{15}$ requires just a simple magnetic system and therefore may be easy to realize. In this paper, the design details of this approach and its simulation results will be given in Secs. II and III, respectively. Afterwards, the tolerance of the efficiency will be investigated in Sec. IV.

\section{BASIC DESIGN}

\section{A. Principle}

The essential part of this design approach is to create a radial drift. In this particular case, an azimuthal electric field component serves to create that drift, as shown in Fig. 1. Generally, a stronger drift implies a larger drift distance $D$ (see Figs. 2 and 6). A large drift distance is preferred for a clean sorting of electron kinetic energy and can provide more tolerance for the beam parameters. The drift velocity

$$
\boldsymbol{v}_{d}=\frac{\boldsymbol{E} \times \boldsymbol{B}}{B^{2}}
$$

and the drift distance $D$ are inversely proportional to the magnitude of the magnetic field. ${ }^{14}$ For a low-frequency gyrotron like a $28 \mathrm{GHz}$ industrial gyrotron, ${ }^{17}$ where the magnetic field is weak, only a small size is required for such an $\boldsymbol{E} \times \boldsymbol{B}$ MDC to obtain enough drift. In that case, the magnetic field in the collector can be kept homogeneous (pure axial) as idealized in Fig. 1. However, for high-frequency fusion gyrotrons, to sustain a homogeneous magnetic field in the collector region is impractical. Therefore, the static stray field of the gyrotron magnet is slightly tuned in the collector region, instead of creating an artificial homogeneous field. An adjusted Single-Stage Depressed Collector (SDC) for the ITER $170 \mathrm{GHz}$ gyrotron ${ }^{1}$ with the tuned magnetic field is taken as the starting point. Figure 3 shows the contour of this SDC. The most important part is the red marked "slope" segment on the collector wall,

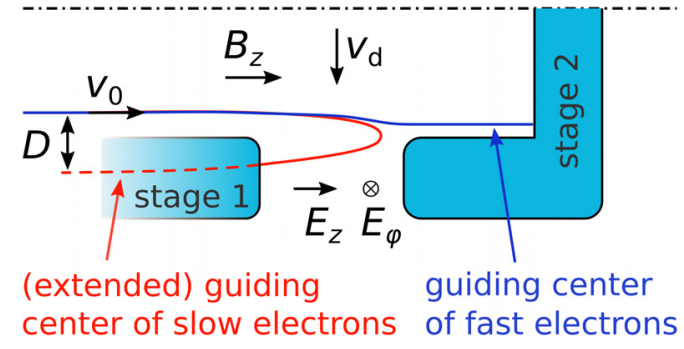

FIG. 2. Principle of the $\boldsymbol{E} \times \boldsymbol{B}$ concept.

to which the magnetic field is aligned locally. Since the electron trajectories follow magnetic field lines in the absence of drifts, the guiding centers of the electrons are parallel to the slope. The electron beam remains several centimeters off the collector wall, while the Larmor radii of the beam electrons are mostly below $3.5 \mathrm{~mm}$ in the entire conic region. Electrons should not impact the wall during the gyration.

Starting from this SDC, a two-stage depressed collector using radial $\boldsymbol{E} \times \boldsymbol{B}$ drift will be conceptually designed. The wall of the SDC is modeled by a thick metallic layer in order to facilitate the mesh generator. Distinct from the multiple electrodes in the similar design, ${ }^{11}$ this paper proposes one single-turn helical slot on the collector wall, which separates the electrodes and introduces an azimuthal electric field component. The torsion of this helix determines the drift strength.

The simplified computer-aided design (CAD) model is shown in Fig. 4. Depending on the properties of the spent electron beam, different depression voltages are applied to each stage. In this example, $-30 \mathrm{kV}$ and $-42 \mathrm{kV}$ depression voltages are chosen, while the kinetic energy of the spent electrons varies from $38 \mathrm{keV}$ to $130 \mathrm{keV}$. Slow electrons up to $42 \mathrm{keV}$ and reflected secondary electrons will be steered by the drift, so that they will impinge the helical cut surface of the first electrode, whereas the fast electrons will travel further and reach the final cylinder bulk, as indicated by Fig. 10 in Sec. III. A local axial sweeping of the electron beam on the cylinder is possible.

The normal-conducting collector coils demonstrated in this example (see Fig. 3) have a maximal $20 \mathrm{mT}$ influence on the original magnetic field inside the collector and can be much shorter than the longitudinal sweeping coils used in the existing gyrotron SDC. Therefore, the winding of these coils should not be a big challenge. The helical gap including the first stage can be enveloped in a vacuum shell.

There is a straight slot joining both ends of the helix for simplicity, shown in Fig. 4(b). This structure causes an opposite drift, which may reflect a tiny fraction of electrons. The reflection of electrons could be significantly minimized, however at the price of increasing the complexity. Part $\mathrm{II}^{26}$ of this work will propose some of such improved designs.

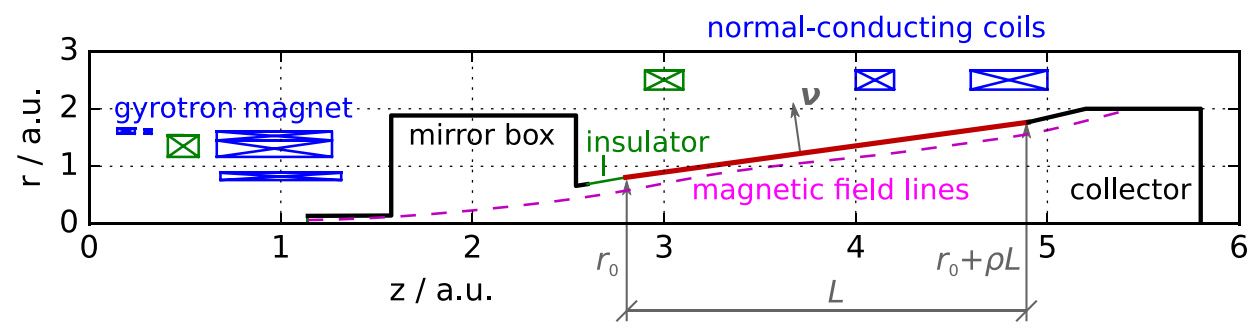

FIG. 3. Contour of a SDC model as the base for the MDC, ${ }^{15}$ (a) cut view and (b) the connection of the helix. 


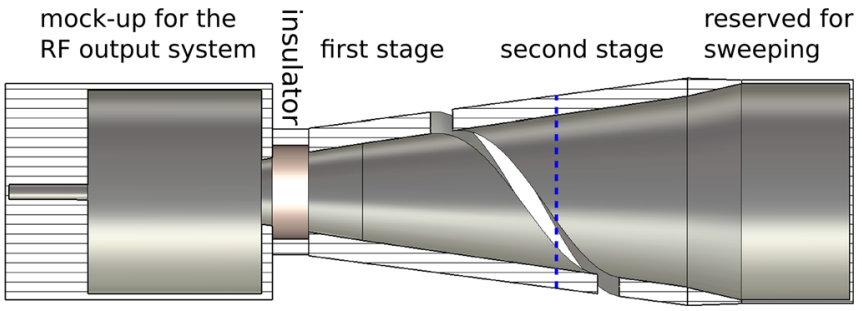

(a)

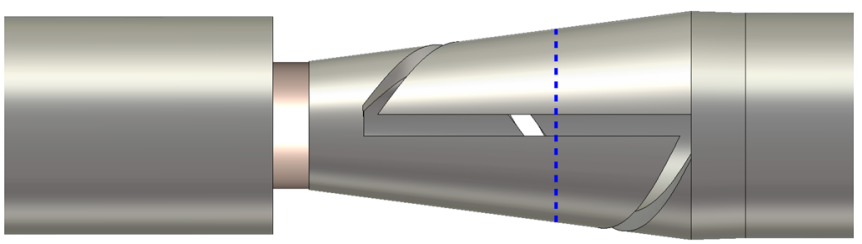

(b)

FIG. 4. Geometry of the new two-stage collector; the marked cross section is presented in Fig. 6.

\section{B. Modeling}

Except the technical limitations on the minimum achievable collector size (such as thermal loading or other technological issues), there is a more fundamental theoretical constraint, from which the minimum size of the collector can be derived. Based on this analysis, the working point is chosen.

The goal is to determine the $\boldsymbol{E} \times \boldsymbol{B}$ drift distance $D$ along the helix, which according to previous work ${ }^{14}$ is

$$
D=\frac{2 v_{0} m_{0}}{B q} \tan \phi=\frac{2 v_{0} m_{0}}{B q} \frac{\sqrt{1-\cos ^{2} \phi}}{\cos \phi}
$$

where $\phi$ is the angle between the electric and magnetic fields. Equation (2) also shows that the drift distance is independent of the magnitude of the electric field in this model. The analysis can be subdivided into four steps: in the first step, a mathematical description of the helix is defined; the second step is an approximate description of the magnetic field; the third step is the calculation of the angle between the fields; finally, all variables will be substituted into (2).

First, the function of a helix curve $\boldsymbol{h}$ from $z=z_{0}$ to $z=z_{0}+L$ has the form

$$
\boldsymbol{h}(z)=\left(h_{x}, h_{y}, z-z_{0}\right)
$$

which should be mathematically simple for any CAD description and will hopefully also be beneficial for the later construction. Let the relative axial displacement be

$$
l:=z-z_{0},
$$

in the range $l \in[0, L]$; one can define the radius $r$ and phase $\varphi$ of the helix on a conical surface as

$$
\begin{gathered}
r=r_{0}+\rho l, \\
\varphi=2 \pi \frac{l}{L},
\end{gathered}
$$

where $L$ is the axial height of the helix, $r_{0}$ is the initial radius, and $\rho \geq 0$ as marked in Fig. 3. Transforming $\boldsymbol{h}$ in Cartesian coordinates yields

$$
\begin{aligned}
& h_{x}=r \cos \varphi=\left(r_{0}+\rho l\right) \cos 2 \pi \frac{l}{L}, \\
& h_{y}=r \sin \varphi=\left(r_{0}+\rho l\right) \sin 2 \pi \frac{l}{L} .
\end{aligned}
$$

Accordingly, the growing direction of the helix is

$$
\boldsymbol{h}^{\prime}=\frac{\mathrm{d} \boldsymbol{h}}{\mathrm{d} l}=\left(\frac{\mathrm{d} h_{x}}{\mathrm{~d} l}, \frac{\mathrm{d} h_{y}}{\mathrm{~d} l}, 1\right) .
$$

Another auxiliary vector is the normal vector of the conical surface, where the helix is located. It has the direction

$$
\boldsymbol{v}=(\cos \varphi, \sin \varphi,-\rho),
$$

which is also marked in Fig. 3.

Second, since the local magnetic field is approximately parallel to the conical surface and free of any azimuthal component (see Fig. 3), the expressions for the components of the magnetic flux density on the cone are

$$
\begin{gathered}
B_{z}=|B| \frac{1}{\sqrt{1+\rho^{2}}}, \\
B_{r}=|B| \frac{\rho}{\sqrt{1+\rho^{2}}}=\rho B_{z} .
\end{gathered}
$$

For the calculation of $|B|$, it is reasonable to assume that the $B_{z}$ would be nearly constant over $r$ ( $\pm 2 \%$ error in this example). According to the flux conservation

$$
B_{z}=\frac{\psi}{\pi r^{2}}
$$

where $\psi$ is the magnetic flux enclosed by a circle with radius $r$, and the magnetic flux density on the wall is

$$
\boldsymbol{B}=\frac{\psi}{\pi r^{2}}(\rho \cos \varphi, \rho \sin \varphi, 1)^{\mathrm{T}} .
$$

Third, in order to calculate the angle $\phi$ in (2), the direction of the local electric field is required. Suppose there is a vector $\mathcal{E}$ aligned with the $\boldsymbol{E}$ field inside the helical cut, i.e.,

$$
\mathcal{E}=a \boldsymbol{E},
$$

for $a \in \mathbb{R}^{+}$. The electric field has to fulfill three conditions:

1. $\mathcal{E}$ is tangential to the surface of the cone, where the helix is located

$$
\boldsymbol{v} \cdot \mathcal{E}=0
$$

2. $\mathcal{E}$ is perpendicular to the helical electrode surface on the first stage, where the slow electrons are supposed to be collected, i.e., perpendicular to the growing direction of the helix

$$
\boldsymbol{h}^{\prime} \cdot \mathcal{E}=0
$$


3. $\mathcal{E}$ points towards the second stage

$$
\mathcal{E}_{z}>0
$$

One solution to these three criteria is

$$
\mathcal{E}=\left(\begin{array}{l}
2 \pi r \rho \cos \varphi+L\left(1+\rho^{2}\right) \sin \varphi \\
2 \pi r \rho \sin \varphi-L\left(1+\rho^{2}\right) \cos \varphi \\
2 \pi r
\end{array}\right)
$$

Finally, the scalar product of (11) and (14) gives the angle between the electric and magnetic fields

$$
\cos \phi=\frac{\mathcal{E} \cdot \boldsymbol{B}}{|\mathcal{E}||B|} .
$$

The drift distance $D$ is calculated by substituting (11) and (15) into (2), which leads to a very lengthy equation. The elegance of this model is that this lengthy expression has an equivalent simple form

$$
D=\frac{m_{0} v_{0} L}{q \psi} r=\frac{m_{0} v_{0} L \rho}{q \psi} l+\frac{m_{0} v_{0} L r_{0}}{q \psi} .
$$

Equation (16) means that the $\boldsymbol{E} \times \boldsymbol{B}$ drift distance increases linearly with the longitudinal offset $l$ of a point on the helix.

Considering the space charge effect and the retarding voltage on the first collector stage, after an electron passes through the first stage into the $\boldsymbol{E} \times \boldsymbol{B}$ region, its velocity $v_{0}$ has the minimum value corresponding to $12 \mathrm{keV}$, according to the simulation. Inserting the minimum of $v_{0}$ into (16), the minimum of the drift distance is obtained. Figure 5 shows the relation of minimum drift distance to electrode radius and collector length.

In principle, the minimum drift distance is preferred to be large. However, a large distance demands a low magnetic field or a large angle between the fields, which correspond to a large radius and long collector geometry, respectively, according to (16).

On the practical side, in order to keep the length of the MDC comparable to a SDC, the length (height) of the cone is fixed at $L=0.9 \mathrm{~m}$ and the maximum radius of the helix should be below $225 \mathrm{~mm}$. This configuration results in a drift distance of around $2 \mathrm{~cm}$ at the beginning of the helix and

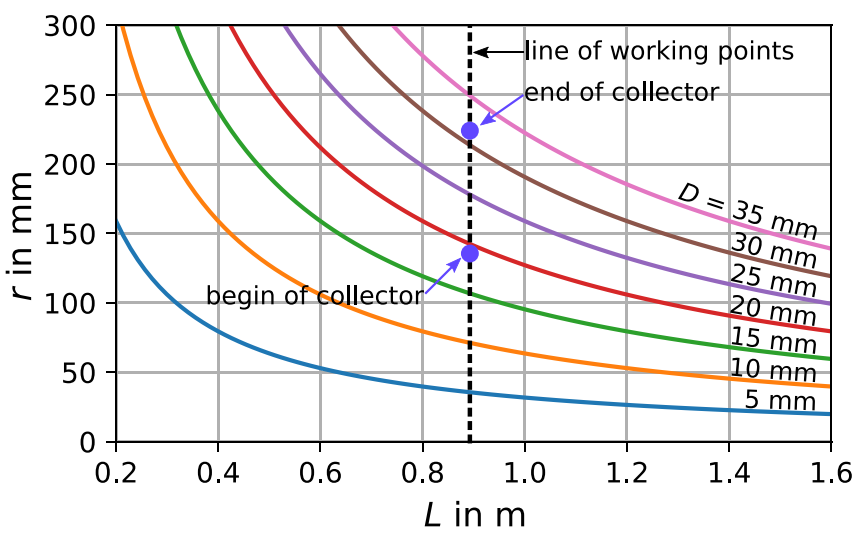

FIG. 5. Minimum drift distance related to the radius and collector length. above $3 \mathrm{~cm}$ at its end. This is the maximum allowed distance between the electron beam and the conical wall, which is controlled by the local magnetic field and indirectly tuned by the current of the auxiliary coils.

\section{SIMULATION}

There are two methods available to simulate MDC models, namely, trajectory TRacKing (TRK) and Particle-In-Cell (PIC). At the time of writing this paper, just a few simulation programs can deal with both methods in such threedimensional irregular models. For the simulation of this particular MDC model, CST Particle Studio is used, where the PIC method implemented in CST does the full electromagnetic wave simulation, rather than only electrostatic. Copper with secondary emission ${ }^{18,19}$ has been built in CST and therefore used in the simulations. The emission model also takes elastic reflections into account.

However, even with recent computational resources, it is still not trivial to simulate this MDC. First, unlike the MDC for the second harmonic X-band gyro-backward-wave oscillator, ${ }^{20,21}$ which has less than $0.25 \mathrm{~T}$ field in the cavity, the $170 \mathrm{GHz}$ ITER gyrotron considered in this model has a 6.77 $\mathrm{T}$ cavity magnetic field. A stronger cavity magnetic field implies a stronger field also in the collector. Neither the conventional concept nor the $\boldsymbol{E} \times \boldsymbol{B}$ drift described in (2) would be effective under a strong magnetic field. In order to reduce the strong magnetic field to a reasonable range, a huge volume is required to dilute the field. As a consequence of the huge $3 \mathrm{D}$ simulation volume, the number of required mesh cells is large in both simulation methods. Second, the injected electron beam has to be sampled with a large number of (macro) sampling electrons, in order to statistically resolve the azimuthal properties of the electron beam. In addition, due to the facts that the simulation volume is large and the electrons are decelerated, it takes a long simulation time (many steps) until (especially the low-energetic) electrons travel through the whole MDC length. With a transient method like PIC, this also means that plenty of injected electrons are within the simulation volume at the same moment. The particle-pushing process can be resource hungry due to the large number of sampled electrons. Third, when an electron impacts the electrode, secondary electrons (including the elastic, inelastic, and the low-energetic true secondary electrons) are emitted from that surface, where the emitted current can be even higher than the impacting one. It will bring additional electrons into the simulation volume and slow down the particle pushing significantly. Depending on the software implementation of the secondary electron model, a large number of electrons have to be injected to get a statistically good sampling of secondary electrons in the 3D MDC model.

Therefore, a compromise has to be made between the computational resources and the accuracy. As it will be shown later, the sampling density of the injected electron beam seems to have more influence on the result quality than the other factors. With the available commercial software and resources, the expected phenomena of this MDC can be observed in the simulation using limited mesh densities. 


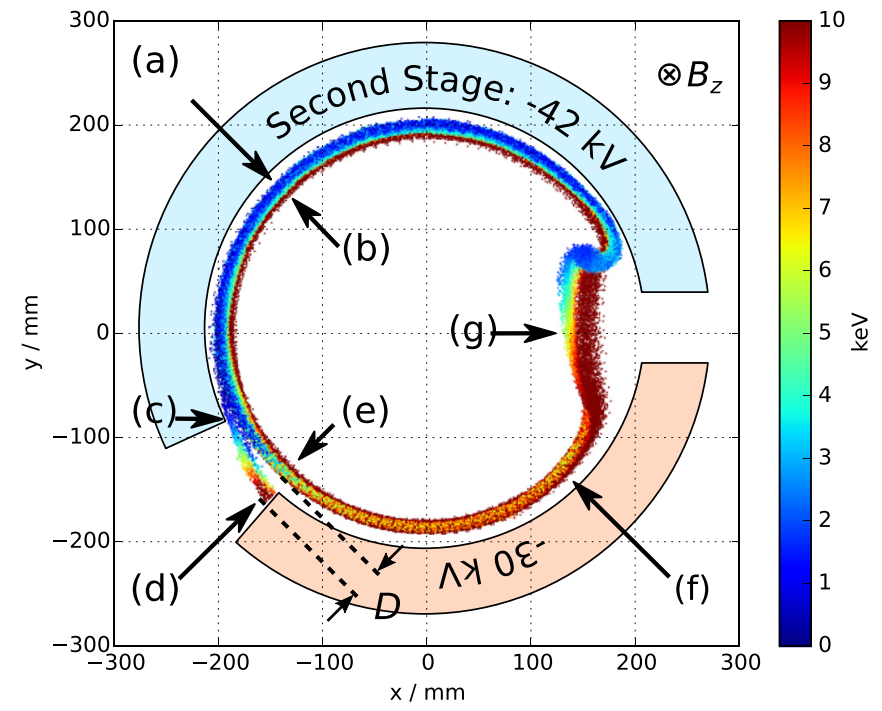

FIG. 6. Cross section of the electron beam in steady-state simulation.

Convergences can be obtained. The results of TRK and PIC methods are in close agreement with each other.

\section{A. Trajectory (TRK) simulation}

With the TRK method, the trajectories of electrons are simulated in multiple iterations. The change of the electric potential or charge (depending on the solver) between two iterations is recorded to consider space charges. In this way, the system may evolve to a steady-state.

Figure 6 shows the electron beam in the cross section marked in Fig. 4. All electrons with kinetic energy higher than $10 \mathrm{keV}$ are colored in red for a better demonstration. The figure depicts the steady-state, rather than a transient moment, which means that all electron spots shown in this diagram belong to the same cut-plane but probably at different temporal moments. It can be observed that one electron appears at this diagram several times because it may be decelerated later and passes through this cut-plane more than one time, like the electrons (d). Even though a small fraction of electrons may be back-and-forth reflected (will be described in the next paragraphs), no electron should be trapped and each iteration of a space-charge calculation stops only when all electrons are collected.

In Fig. 6, there is a gap between (c) and (d). The gap belongs to the curling helical slot. The azimuthal location of the gap in this diagram depends on the axial displacement of the cross section. Electrons pointed by (a) and (b) have lost kinetic energy, but they were too fast to enter the gap at that azimuthal angle where they are. Therefore, these electrons were not collected by the first electrode and can move further until the end of the collector. The slower electrons are on the outer side (a), whereas the faster electrons are on the inner side (b) because the slower electrons are exposed longer in the radial drift region. The electrons, which will be collected on the first stage, are first decelerated and then backwards accelerated during their drift towards the helical gap (see also the white trajectory in Fig. 7). Electrons pointed by (c) in Fig. 6 are under deceleration, whereas those pointed by (d) are the ones which are accelerated backwards to the first stage. Since this diagram is a cross section in the case of steady-state and the drift direction is radial, the electrons pointed by (d) are the same ones under (e). (f) points the electrons which have not been influenced by the azimuthal electric field yet. Those electrons pointed by $(\mathrm{g})$ are at the specific angle where the drift has an opposite direction. The slow electrons at this position cannot be optimally collected and are discussed in the next paragraphs.

Figure 7 shows some representative electron trajectories and the steady-state of the electric potential in the simulation region, while taking space charges of both primary and secondary electrons into account. A typical low-energetic electron has the white trajectory. It is collected on the helical surface of the first stage, whereas the yellow ones are the high-energetic electrons, which are collected on the second stage, as expected. The magenta trajectory represents one of the electrons, which is on the specific azimuthal angle. In this particular example, the magenta electron at this angle encounters an undesired drift towards the center and does not have enough longitudinal velocity to land on the second stage; therefore, it turns back to the mirror box with enlarged cyclotron motion. This kind of reflected electron has only a small chance to pass through the compressing magnetic field in the mirror box because of the nonreciprocal increment of cyclotron motion; more probably, it will turn back and enter the MDC again at a different azimuthal angle. The change of angle is the consequence of azimuthal drifts. At another angle, some of these electrons overcome the potential barrier caused by the retarding voltage and the asymmetric space charges. These electrons will be collected either on the second stage like in this example or on the first stage.

Although this concept-approval basic design is simple and promising, it is not flawless. There will be a small

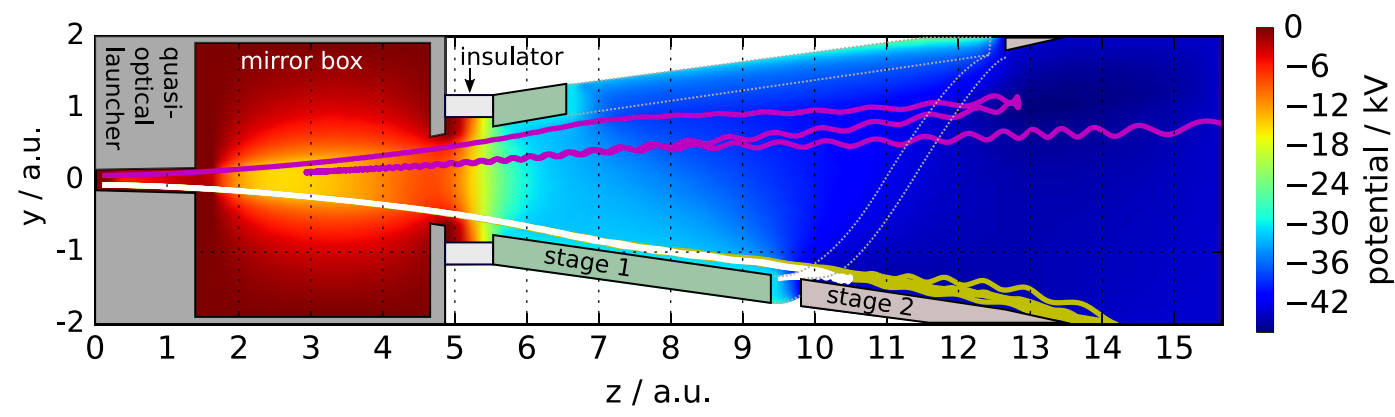

FIG. 7. Electrical potential considering space charge (including that of secondary electrons) in the $z$-y-plane and some representative electron trajectories. 
fraction of electrons which return to the quasi-optical launcher or even travel backwards into the cavity. They are the slow electrons injected at the azimuthal angle of the straight cut between the stages. Some of these electrons move back and forth for one or multiple times and are finally collected by an electrode at another azimuthal angle. Only a small fraction of them can reach the launcher. Any electron that reaches the launcher is counted as loss in the later calculation of the collector efficiency. In this example, the finally reflected current (including the current of reflected secondary electrons) is according to the simulation $700 \mathrm{~mA}$, which equals approximately $1.5 \%$ of the primary injected current $45 \mathrm{~A}$. These electrons will probably participate in the interaction again. They will eventually gain energy from the RF and will be mainly ${ }^{2}$ collected by the collector. There are reasons to believe that this quantity of reflection can be harmless. In the literature, ${ }^{2}$ only $10 \mathrm{~mA}$ reflection is measured while the simulation predicted a gross reflected current of $640 \mathrm{~mA}$, which is in the same order as with the presented MDC. The reflected electrons may or may not influence the interaction. In the experiment of the SDC for a $140 \mathrm{GHz}$ gyrotron, ${ }^{3}$ where $I_{\mathrm{b}}=27 \mathrm{~A}$ and $U_{\mathrm{b}}=80 \mathrm{kV}$; the output $\mathrm{RF}$ power did not change, even when the measured reflected current has reached $3 \mathrm{~A}$. Further investigations are necessary on the impact of the $700 \mathrm{~mA}$ reflected current, in particular on the thermal loading and potential issues of the power supply. Optimization like reducing the width of the straight slot, so far the field does not break down, will slightly reduce the reflected current. There are effective methods to significantly reduce the $700 \mathrm{~mA}$ reflection. They will be presented in Part $\mathrm{II}^{26}$ of this work.

The sampling of electrons influences the convergence of TRK iterations the most. Figure 8 shows the accuracy convergence of the TRK solver over iterations. The logarithmic accuracy is defined as

$$
\delta_{\mathrm{dB}}=20 \log _{10} \frac{\left|\boldsymbol{q}_{i+1}-\boldsymbol{q}_{i}\right|}{\left|\boldsymbol{q}_{i+1}\right|},
$$

in CST, where $\boldsymbol{q}_{n}$ is the charge distribution in the $n$-th iteration. The noise level in the TRK convergence is the combined results mainly from two aspects:

- The small fraction of reflected electrons by the opposite drift may cause changes of space charges in each iteration, unless there is a dense sampling of electrons at the specific angle to present the space charge statistically accurate.
- Secondary electrons are numerically hard to sample within limited computational resources. The common model for the secondary emission has to limit the number and generation of secondary electrons so that a few secondary electrons that are emitted at random angles with random initial energy have to delegate the "cloud" of secondary electrons in the real world. The randomness brings extra noise.

Three pairs of simulations having the same design and mesh are compared. Each pair contains the realistic simulation (solid line) and the variant without secondary electron (dashed line) as a reference simulation. In this way, the contributions of the two kinds of noise sources can be distinguished.

The only difference between the pairs of simulation setups is the number of injected electrons. Generally, the denser the electrons are sampled the lower will be the noise. After several iterations, the decrement of the charge difference slows down. The distances between the dashed lines show about $10 \mathrm{~dB}$ convergence improvement per 10 times of the number of electrons. If the distributions of the electrons in the special angle are well resolved (the $10 \mathrm{k}$ and $100 \mathrm{k}$ cases), the randomness of secondary electrons adds another $5 \mathrm{~dB}$ to the convergence noise.

The TRK solver shows a collector efficiency of $\eta_{\mathrm{col}}$ $=79 \%$ in the absence of secondary electrons and $78 \%$ if taking secondary electrons into account. This collector efficiency should be sufficient to increase the overall gyrotron efficiency above $60 \%$.

\section{B. Verification with full-wave particle-in-cell (PIC)}

In order to verify the steady-state TRK results, this MDC model is cross-checked with full-wave PIC simulations, which are more time and memory consuming than the TRK method. The PIC simulation is transient. After several hundreds of nanoseconds, the system may reach a steady state but with a lot of noise. Like the observation in the TRK simulations, noise is mainly introduced by the statistical sampling of the primary and secondary electrons.

Figure 9 shows an example of the PIC convergence compared with the TRK result. The impacted current in this diagram consists of the primary electron beam as well as the multiple times collected secondary electrons. The same MDC model is investigated within two simulations. The quantity of injected electrons at each time step is the only difference between both simulations. In the first simulation, one thousand electrons are injected at each time step (the

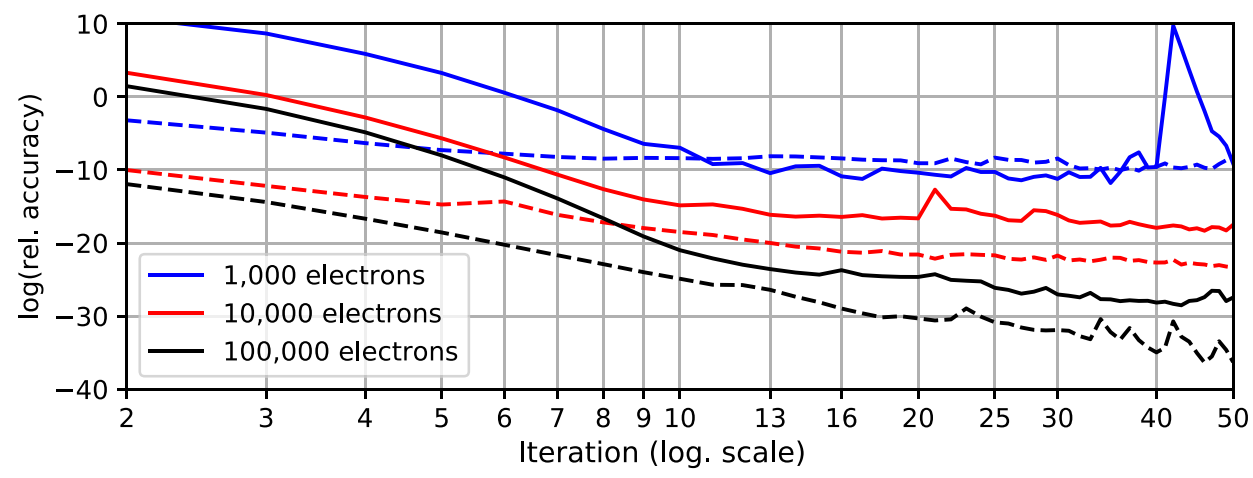

FIG. 8. Relative TRK iteration accuracy, solid lines take secondary electrons into account, while dashed lines do not. 


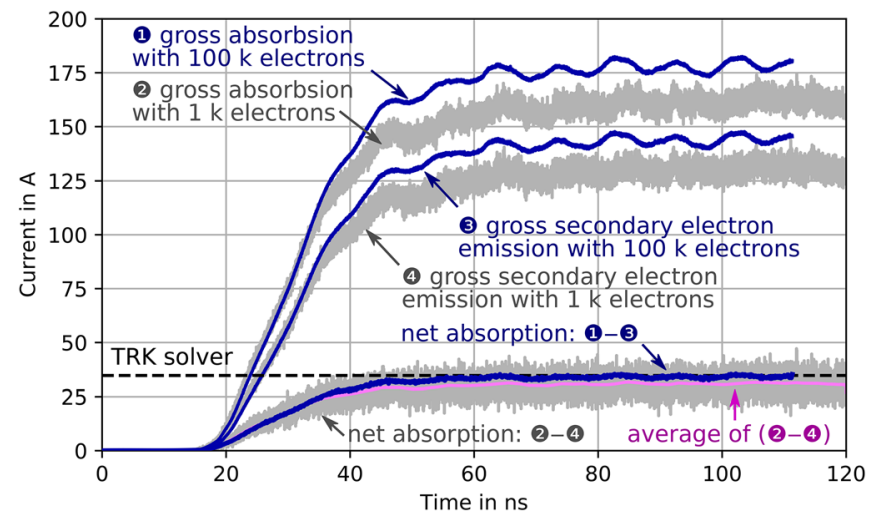

FIG. 9. PIC convergence related to the number of sampling electrons, taking the current on the second stage as an example.

gray curves in Fig. 9). These electrons are distributed quasihomogeneously around the azimuthal angle. In the second simulation (the blue curves in Fig. 9), the same electron beam is sampled with 100000 macro-electrons each time step, while keeping the total injected current unchanged. Since the latter electron beam has a better statistical representation of the beam, the noise observed in the simulation is many magnitudes lower. In addition, the more the primary electrons impact electrodes, the finer the secondary electrons would be sampled and the more precise the statistic model would be. For this reason, both impacted and emitted current of the fine simulation case are higher than in the coarse simulation. In spite of the difference in the absolute values, both configurations have their (average) net current $\left(I_{\text {impact }}-I_{\text {emit }}\right)$ at the same level, even though the current in the coarse simulation is more noisy.

Because the spent electron beam of gyrotrons has a broad energy spectrum and is injected from the launcher, a fast electron in the beam may need only tens of nanoseconds to travel through the entire collector region up to the second stage, while at this moment, the slow electrons may not yet pass the mirror box. Therefore, the transient current on the second collector stage evolves faster into a steady state than on the other parts of the MDC. The fine simulation stopped at $110 \mathrm{~ns}$ limited by the computational resources. Fortunately, this duration is already enough to drive the current on the second stage into a steady state, as shown in Fig. 9. To compare the net current in both cases, the average value for the case with one thousand electrons is calculated. In the steady state, the average current is not far away from the TRK result, whereas with a denser sampled electron beam (i.e., the 100 thousand cases), the current predicted by the PIC and TRK methods is in close agreement. Concerning the limitation of the computational resources, the coarse PIC simulation will be evaluated.

Figure 10 is a snapshot of the electron position in a hollow beam during the PIC simulation. It has the same view as Fig. 4(a). The colors of electrons stand for their kinetic energy, with red for high energy and blue for low energy. The whole electron beam is decelerated by $30 \mathrm{kV}$ immediately after it enters the collector. Then, the helical electrode splits the low-energetic electrons out. The sparse electrons that appear on the end of the second stage are mostly the slow-moving secondary electrons.

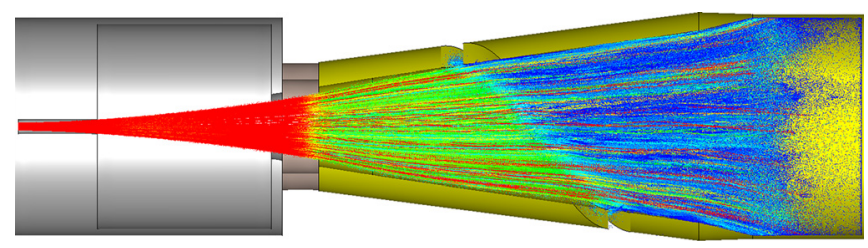

FIG. 10. Electron positions (including secondary electrons) in an arbitrary transient time step of PIC simulation.

In Fig. 11, all net currents are shown. The simulation takes secondary electrons into account. The orange lines are the average value of each noisy curve. As expected, the average current on the second stage stabilized inside the first $100 \mathrm{~ns}$, whereas the whole system evolves into a steady state $50 \mathrm{~ns}$ later, due to the slow electrons. The average value of the total collected current in the steady state is in perfect agreement with the expected $45 \mathrm{~A}$ injected current (dashed line), which means that no electron is trapped.

Finally, the efficiency of the collector is shown in Fig. 12. A collector efficiency of $77 \%$ in average is observed in the PIC simulation, which considers the effects of secondary electrons, whereas in the absence of secondary electrons, the MDC performs only $1 \%$ better. This means that this kind of MDC handles secondary electrons well. The predicted efficiency by PIC simulation is in agreement with the TRK result.

\section{Thermal loading}

On the second stage, the decelerated electron beam can be axially swept; moreover, cooling a cylindrical surface is perhaps easier than cooling the first stage, which is on a helical surface with the gradually increasing radius. Therefore, more attention has been paid to the cooling demand of the first stage. However, obtaining the thermal loading of such an irregular 3D surface can be tricky using the currently available simulation tools.

Due to the limitation of the software implementation at the time of writing this paper, the thermal loading is calculated by CST in power per volume. However, it makes more sense to obtain the local value of the collected beam power per area, which has not been implemented in CST yet. Neither is the analysis of exported particle trajectories an option, because of the lack of internal information from the commercial software. After all, since the collected power on any object is obtainable, a work around would be overlaying

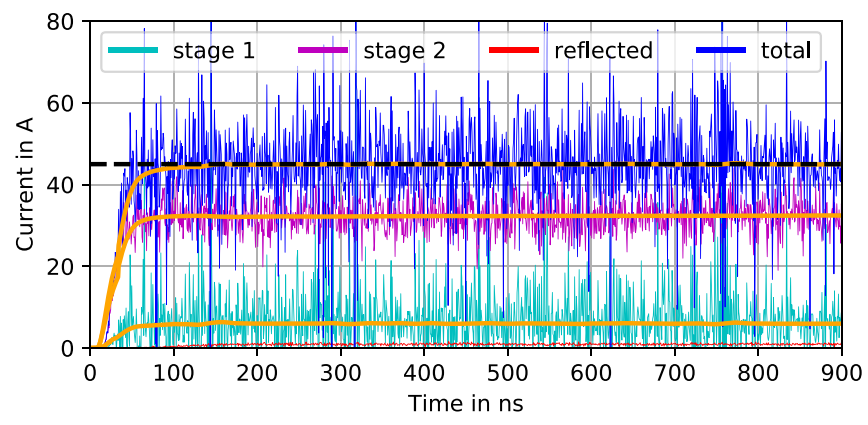

FIG. 11. Transient currents from the PIC simulation, ${ }^{22}$ their average values (orange), and the desired total current (dashed). 


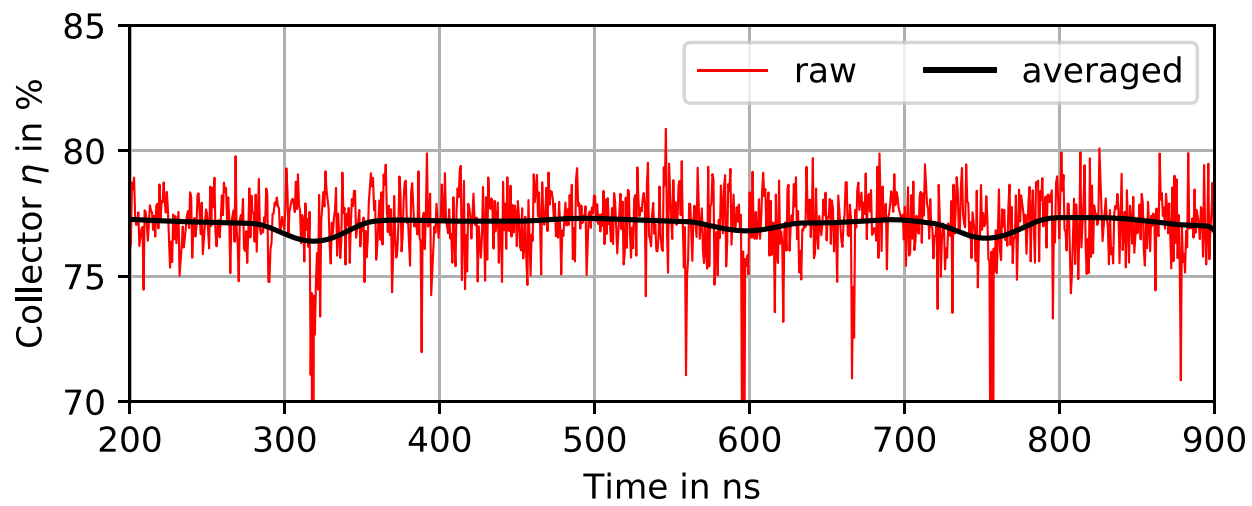

FIG. 12. Collector efficiency considering secondary electrons. ${ }^{22}$

a thin layer above the helical surface of the electrode. This thin layer consists of plenty of small mesh fragments, each of which is an individual object. The collected power of each fragment can be obtained from a simulation and the area of its surface is known; thus, the power per local area information is calculable. This method only provides quite rough information, but at least it is now capable of estimating an approximation of the thermal loading.

To plot the thermal loading of the curved helical electrode surface in a figure, the surface is projected in the transversal plane, which maps the mesh fragments to sectors. The power density is treated as a constant over each fragment. Figure 13 shows the distribution of power density on the sectors. The power is concentrated just on the inner edge of the helical surface, and there is no leakage of current observed in the simulation. This means that the choice of the helix thickness is conservative and the electrode can be narrower and compacter than in this example. The transition of colors was expected to be more gradual, probably because of the noise mentioned in Secs. III A and IIIB (which is hard to prove under the current resource and software implementation), and

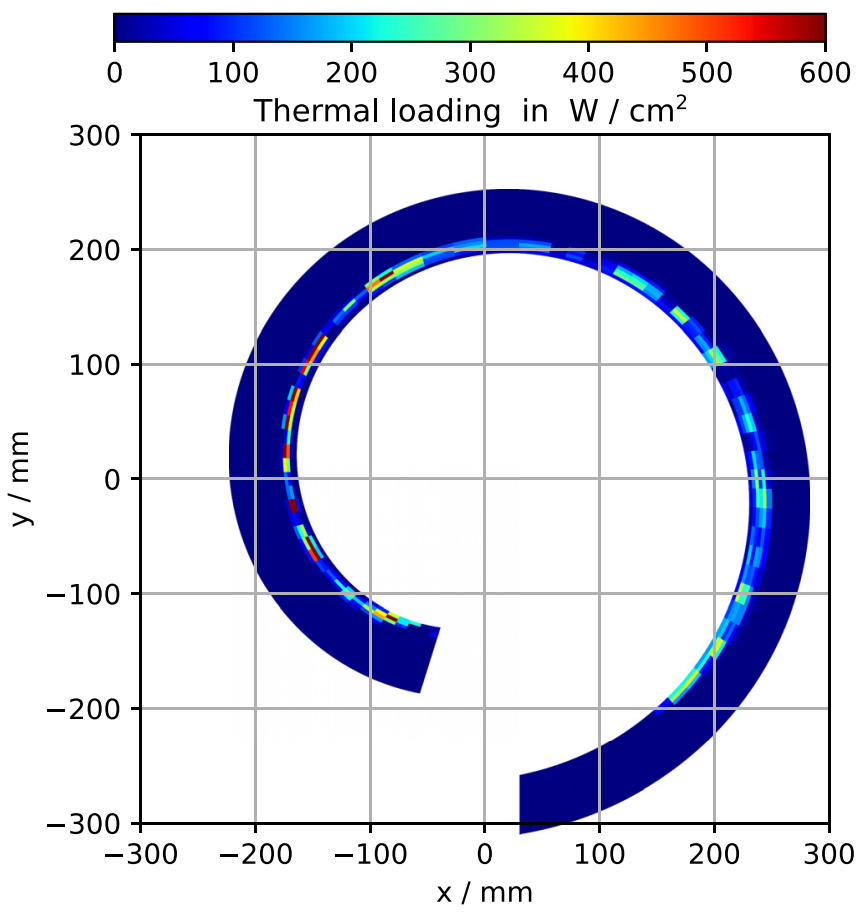

FIG. 13. Thermal loading on the surface of the first collector stage. the level of power density between two adjacent fragments can be sometimes quite different. Moreover, the noise may cause some hot spots in the region with high loading. Most fragments have thermal loading below $400 \mathrm{~W} / \mathrm{cm}^{2}$ in the steady state. The region near the entrance of the collector (negative $x$ in the diagram) has somewhat higher loading than the region near the end of the collector. The reason is that at the entrance of the collector, the helix has a smaller radius than at the end; in addition, the electron beam is also more focused at the entrance. Hence, the density of impacting electrons is higher at the entrance. If the area of a fragment is tiny, even a single impacted (macro) electron would cause significant power density. As in this diagram, each fragment is radially less than $3 \mathrm{~mm}$ wide. The oddly high loading will vanish, if the "hot" fragments are averaged with the neighboring ones, becoming $6 \mathrm{~mm}$ fragments. Therefore, it is tricky to present the surface power density statistically accurate. The torsion of the helix could be tuned to achieve a homogeneous thermal loading profile. Nevertheless, due to the capability of the available simulation software, no further optimization step has been taken regarding the thermal loading.

\section{TOLERANCE}

$\boldsymbol{E} \times \boldsymbol{B}$ drift sorts electrons reliably, since the sorting depends on the electric potential of the stages rather than the local electric field. The sorting relies on neither the space charge repulsion nor the divergence of the magnetic flux. Besides, the moderate magnetic field inside the collector still confines and stabilizes the electron beam (in this example: $60 \mathrm{mT}$ at the entrance, while $15 \mathrm{mT}$ at the end). Therefore, this sorting mechanism should be stable to the variation of beam current (space charge) and does not require the magnetic field to be highly precise at low magnitude; the latter one also means that this kind of MDC may tolerate undesired perturbation of the magnetic field. In this section, the performance of this MDC will be investigated, in particular on three effects:

1. influence of the beam current, which is related to the space charge and repulsion;

2. undesired magnetic field perturbations, which come from the stray fields of the fusion tokamak and neighboring gyrotrons;

3. misalignment of the electron beam. 
These may influence the performance of a conventional gyrotron MDC.

To investigate the MDC performance regarding a variable beam current, simulations are performed with only the beam current being scaled in a range of $\pm 20 \%$, while keeping the kinetic spectrum of the electron beam and the retarding voltages of the MDC unchanged. Figure 14 presents the collected power over the beam current from the simulations, considering repulsion as well as space charges. The collected beam power follows the beam current linearly. Moreover, the ratio between both is almost unity, which means that the performance of this MDC does not rely on the beam current.

From tokamak and neighboring gyrotrons, there could be a stray magnetic field, which may influence the performance of a single or multi-stage depressed collector. As presented in the literature, ${ }^{23,24} 5 \mathrm{G}$ is a reasonable value to be considered for the stray magnetic field from the environment. Based on the simulation in Sec. III, an additional homogeneous transversal magnetic field with variable azimuthal angles is applied in the entire collector region. Figure 15 shows the efficiency of this MDC in an external perturbation magnetic field, where the direct path connecting the ends of the helix is located at $0^{\circ}$. In order to keep the time duration for the simulations acceptable, secondary electrons are not considered. Since the MDC geometry and its electric field are not axisymmetric, the efficiency diagram is also expected to be anisotropic. In the worst case of this example, the twostage collector still has $73 \%$ collector efficiency under a $5 \mathrm{G}$ transversal stray magnetic field, which nearly accomplishes the desired value of $74 \%$ for the goal $\eta_{\text {total }}>60 \%$. If the collector could be rotated in the optimum orientation, its efficiency would not be reduced by the external magnetic field.

The electron beam could have up to $0.5 \mathrm{~mm}$ displacement in cavity, still allowing single-mode oscillation. ${ }^{25} \mathrm{~A}$ realistic MDC should be capable of tolerating this misalignment. The tolerance is again checked by simulations, where only the injecting position of the electron beam should be shifted, while keeping the magnetic field and the geometry always centered. Even though this method is not strictly correct (a misaligned electron beam would have slightly different energy and pitch factor distributions), it is acceptable for the demonstration. The beam misalignment in the cavity is approximately converted to the one in the injection position via

$$
\Delta r_{\text {inj }}=\sqrt{\frac{B_{\text {inj }}}{B_{\text {cavity }}}} \Delta r_{\text {cavity }},
$$

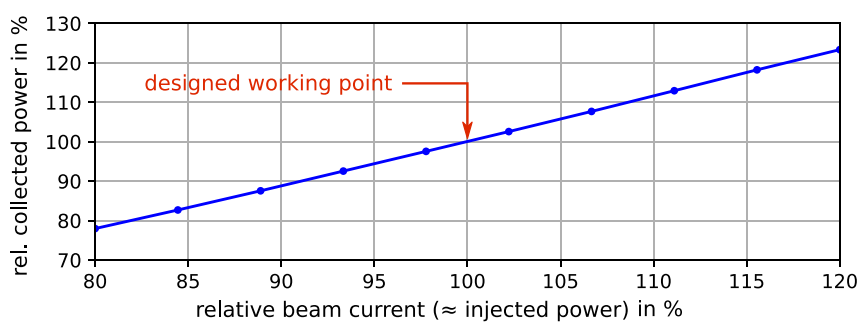

FIG. 14. Influence of beam current on the collected power.

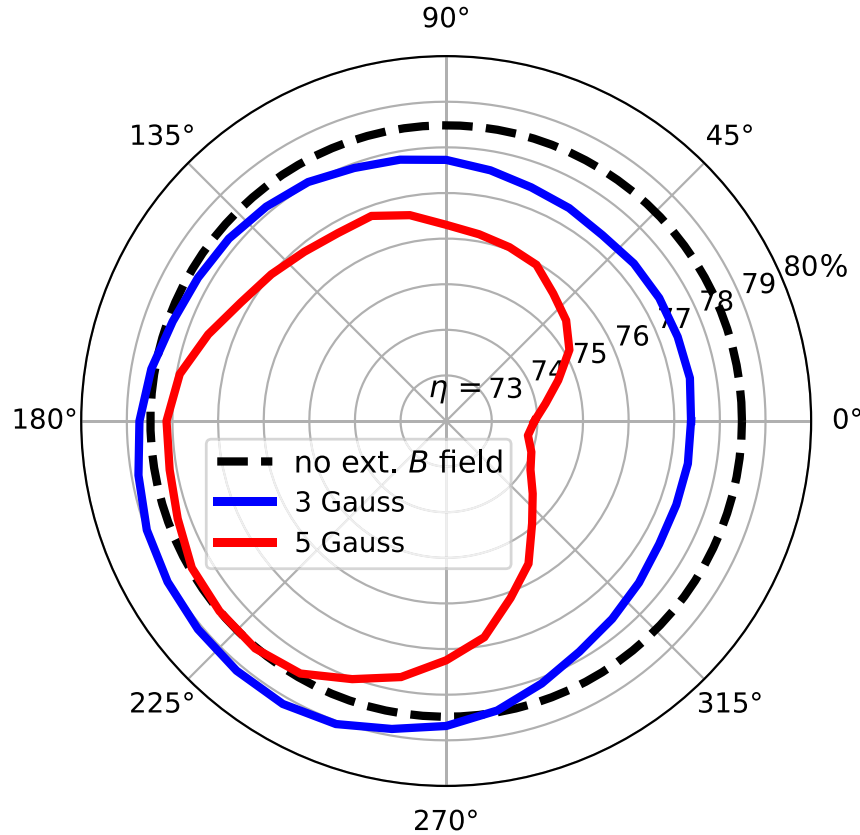

FIG. 15. Collector efficiency in an external magnetic field.

based on the flux conservation. For instance, $0.5 \mathrm{~mm}$ beam offset in the cavity of the $170 \mathrm{GHz}$ gyrotron causes 20 times more offset at the end of the collector. This will challenge the conventional MDC concept, which has to use a low magnetic field in order to perform well, whereas for this $\boldsymbol{E} \times \boldsymbol{B}$ concept, there is no problem at all, as shown in Fig. 16. Even a larger offset up to $0.8 \mathrm{~mm}$ would not cause any significant reduction of its performance.

Besides the collector efficiency, tolerances on the reflected electron trajectories and changes of the power loading are also important. They are not considered in this simulation but must be addressed before the capability of this MDC concept is determined.

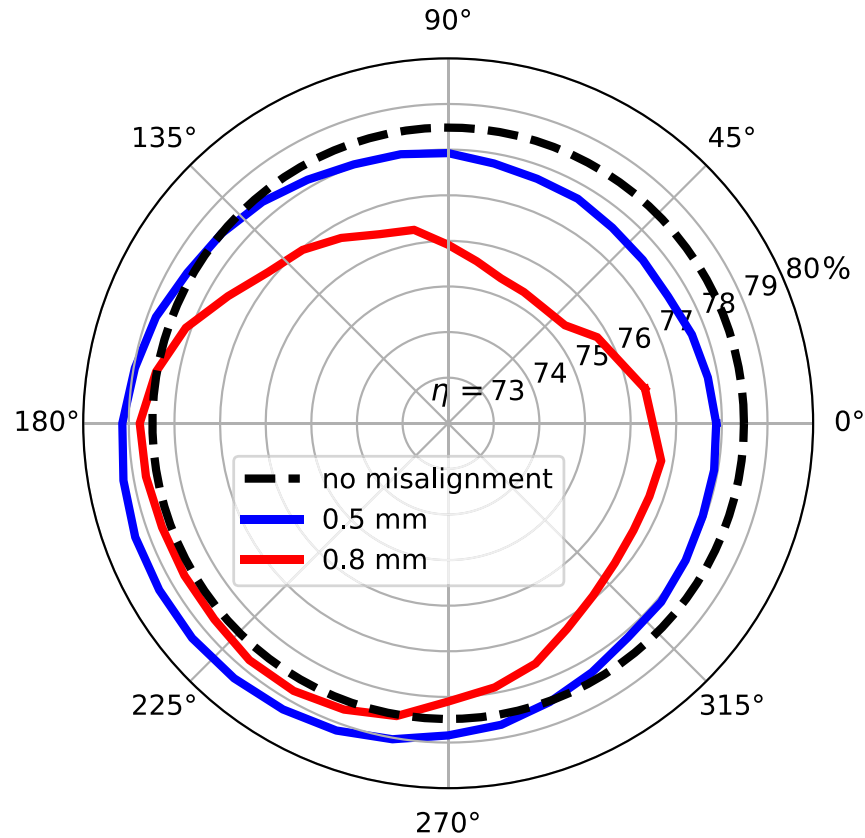

FIG. 16. Collector efficiency with the misaligned electron beam in the cavity. 


\section{CONCLUSIONS}

The computational design of a feasible and promising gyrotron MDC is presented. In this design, a radial $\boldsymbol{E} \times \boldsymbol{B}$ drift is created by the azimuthal electric field and longitudinal magnetic field, where the electric field is maintained by a pair of helical electrodes. Utilizing the $\boldsymbol{E} \times \boldsymbol{B}$ drift, this kind of MDC sorts electrons reliably, and hence, it has the potential to achieve very high collector efficiency. An example of a two-stage collector for the EU $170 \mathrm{GHz}, 1 \mathrm{MW}$ ITER gyrotron was simulated using a realistic spent electron beam. This two-stage collector is able to increase the gyrotron overall efficiency from $50 \%$ to more than $60 \%$. The efficiency reduction by secondary electrons (including the elastically and inelastically reflected ones) is insignificant, due to the facts that first, the secondary electrons are exposed in the same $\boldsymbol{E} \times \boldsymbol{B}$ drift like the primary ones and are guided back to the first electrode; second, there is in principle no local accelerating electric field which backstreams the secondary electrons from the second stage.

The shape and size of this kind of collector can be approximately modeled using simple equations. Both trajectory-tracking and particle-in-cell solvers built in CST Particle Studio are used against each other to verify the design. Their convergences are presented, and the results of both methods are in close agreement. Simulations also indicate that beam current, misalignments of the electron beam, and perturbations in the magnetic field do not influence the collector efficiency a lot.

However, there is a straight slot joining the ends of the helical electrodes together. It simply separates the collector stages but introduces an undesired drift towards the center axis. A small part of low energetic electrons can be reflected due to this drift. In this specific design example, $1.5 \%$ of the beam current is reflected. Even though the ratio of reflected current can be reduced via parameter optimizations, there are more effective ways to minimize the reflection. Such enhancements will be handled in Part $\mathrm{II}^{26}$ of this work.

To further extend this basic design in the future, the MDC could have more than two stages by stacking helical electrodes longitudinally. Besides, there can be multiple helices dividing the azimuthal circle into sectors, instead of one helix curling $360^{\circ}$. This change can shrink collector length as well as bring other advantages. The torsion of the helix can be optimized, too.

\section{ACKNOWLEDGMENTS}

This work was carried out within the framework of the EUROfusion Consortium and has received funding from the Euratom research and training program 2014-2018 under Grant Agreement No. 633053. The views and opinions expressed herein do not necessarily reflect those of the European Commission.
The authors thank Professor E. Borie for the contribution to the text quality of this manuscript.

${ }^{1}$ J. Jelonnek, S. Alberti, K. Avramidis, V. Erckmann, G. Gantenbein, K. Hesch, J.-P. Hogge, S. Illy, J. Jin, S. Kern, I. Pagonakis, B. Piosczyk, T. Rzesnicki, A. Samartsev, and M. Thumm, Fusion Sci. Technol. 64, 505 (2013).

${ }^{2}$ K. Sakamoto, M. Tsuneoka, A. Kasugai, T. Imai, T. Kariya, K. Hayashi, and Y. Mitsunaka, Phys. Rev. Lett. 73, 3532 (1994).

${ }^{3}$ B. Piosczyk, C. T. Iatrou, G. Dammertz, and M. Thumm, IEEE Trans. Plasma Sci. 24, 579 (1996).

${ }^{4}$ M. Y. Glyavin, A. Kuftin, N. Venediktov, and V. Zapevalov, Int. J. Infrared Millimeter Waves 18, 2129 (1997).

${ }^{5} \mathrm{C}$. Wu, I. Pagonakis, S. Illy, M. Thumm, G. Gantenbein, and J. Jelonnek, in German Microwave Conference (GeMiC) (2016), pp. 365-368.

${ }^{6}$ T. Okoshi, E.-B. Chiu, and S. Matsuki, IEEE Trans. Electron Devices 19, 104 (1972)

${ }^{7}$ H. G. Kosmahl, IEEE Proc. 70, 1325 (1982).

${ }^{8}$ A. Singh, S. Rajapatirana, Y. Men, V. Granatstein, R. Ives, and A. Antolak, IEEE Trans. Plasma Sci. 27, 490 (1999).

${ }^{9}$ G. Ling, B. Piosczyk, and M. K. Thumm, IEEE Trans. Plasma Sci. 28, 606 (2000).

${ }^{10}$ M. Y. Glyavin, M. V. Morozkin, and M. I. Petelin, Radiophys. Quantum Electron. 49, 811 (2006).

${ }^{11}$ I. G. Pagonakis, J. P. Hogge, S. Alberti, K. A. Avramides, and J. L. Vomvoridis, IEEE Trans. Plasma Sci. 36, 469 (2008).

${ }^{12}$ I. G. Pagonakis, C. Wu, K. A. Avramidis, G. Gantenbein, S. Illy, M. Thumm, and J. Jelonnek, in Proceedings of the 42th International Conference on Infrared, Millimeter and Terahertz Waves (IRMMW-THz) (2017).

${ }^{13}$ I. G. Pagonakis, C. Wu, S. Illy, and J. Jelonnek, Phys. Plasmas 23, 043114 (2016).

${ }^{14}$ C. Wu, I. G. Pagonakis, G. Gantenbein, S. Illy, M. Thumm, and J. Jelonnek, Phys. Plasmas 24, 043102 (2017).

${ }^{15}$ C. Wu, I. G. Pagonakis, S. Illy, G. Gantenbein, M. Thumm, and J. Jelonnek, in 2017 IEEE International Vacuum Electronics Conference (2017), pp. 1-2.

${ }^{16}$ O. I. Louksha and P. A. Trofimov, Tech. Phys. Lett. 41, 884 (2015).

${ }^{17}$ A. Malygin, S. Illy, I. G. Pagonakis, B. Piosczyk, S. Kern, J. Weggen, M. Thumm, J. Jelonnek, K. A. Avramides, R. L. Ives, D. Marsden, and G. Collins, IEEE Trans. Plasma Sci. 41, 2717 (2013).

${ }^{18}$ M. A. Furman and M. T. Pivi, Phys. Rev. Spec. Top. Accel. Beams 5, 124404 (2002).

${ }^{19}$ M. A. Furman and M. T. F. Pivi, Phys. Rev. Spec. Top. Accel. Beams 16, 069901 (2013).

${ }^{20}$ L. Zhang, W. He, A. W. Cross, A. D. R. Phelps, K. Ronald, and C. G. Whyte, IEEE Trans. Plasma Sci. 37, 2328 (2009).

${ }^{21}$ W. He, K. Ronald, A. R. Young, A. W. Cross, A. D. R. Phelps, C. G. Whyte, E. G. Rafferty, J. Thomson, C. W. Robertson, D. C. Speirs, S. V. Samsonov, V. L. Bratman, and G. G. Denisov, IEEE Trans. Electron Devices 52, 839 (2005).

${ }^{22}$ C. Wu, I. G. Pagonakis, G. Gantenbein, S. Illy, M. Thumm, and J. Jelonnek, in Proceedings of the 42th International Conference on Infrared, Millimeter and Terahertz Waves (IRMMW-THz) (2017), pp. $1-2$.

${ }^{23}$ I. G. Pagonakis, F. Li, S. Illy, B. Piosczyk, S. Alberti, J.-P. Hogge, S. Kern, M. Henderson, and C. Darbos, IEEE Trans. Plasma Sci. 40, 1945 (2012).

${ }^{24}$ B. Ell, I. G. Pagonakis, G. Gantenbein, S. Illy, M. Thumm, and J. Jelonnek, IEEE Trans. Electron Devices 64, 3421 (2017).

${ }^{25}$ A. Samartsev, G. Dammertz, G. Gantenbein, J. Jelonnek, S. Illy, and M. Thumm, IEEE Trans. Plasma Sci. 41, 872 (2013).

${ }^{26}$ C. Wu, I. G. Pagonakis, K. A. Avramidis, G. Gantenbein, S. Illy, M. Thumm, and J. Jelonnek, Phys. Plasmas (in preparation). 